RU96-15-B

\title{
SYMMETRIES OF 2-D GRAVITY
}

\author{
Ioannis Giannakis ${ }^{(a)} \dagger$ \\ (a) Physics Department, Rockefeller University \\ 1230 York Avenue, New York, NY 10021-6399
}

\begin{abstract}
Two-dimensional gravity in the light-cone gauge was shown to exhibit an underlying $\operatorname{sl}(2, R)$ current algebra. It is the purpose of this note to offer a possible explanation about the origin of this important algebra. The essential point is that two-dimensional gravity is governed by a topological field theory. The gauge group is $\operatorname{sl}(2, R)$ and it is this enhanced gauge group that yields Polyakov's current algebra.
\end{abstract}

$\dagger$ e-mail: giannak@theory.rockefeller.edu 


\section{Introduction.}

Gravity in two-dimensional space-times has received extensive scrutiny in recent years, both because of its importance in string theory, where it plays a crucial rôle in world-sheet dynamics, and as a tractable toy model for a quantum theory of gravity in higher dimensions. As is usually the case for an effective toy model, the dynamics of two-dimensional gravity is just non-trivial enough to be interesting, while remaining largely tractable.

At first sight, two-dimensional general relativity would appear to be a too trivial topological theory, since the Einstein-Hilbert Lagrangean density is a total divergence, and the space-time metric is a gauge artifact devoid of classical dynamics. Quantum mechanically, of course, this is not true; except when coupled to conformal matter with an appropriate central charge, quantum effects induce non-trivial dynamics for the metric modulo coordinate transformations. In this way, we are led naturally to versions of twodimensional scalar-tensor gravity in which non-trivial equations of motion specify the scalar curvature to be a (cosmological) constant. In conformal gauge this leads to Liouville theory, but much greater progress has come from Polyakov's introduction of lightcone gauge, in which the only degree of freedom is the $g_{++}$component of the metric. This is a natural gauge choice because $\operatorname{det} g=1$, and all geometrical quantities (Christoffel symbols, curvatures) are polynomial in $g_{++}$and its derivatives. However, the real power of this gauge choice lies in Polyakov's discovery of an $s l(2, R)$ current algebra within the algebra of observables [1]. It is this current algebra that makes the theory tractable, but its appearance would appear to be entirely mysterious - unmotivated by any principle that goes into the formulation of the theory. Several proposals towards unraveling its origin have been made in the literature [2]. It is our purpose in this note to offer an alternative explanation about the origin of this important algebra.

The essential point is that, despite appearances, this theory too is governed by a topological field theory, this time of Chern-Simons type (i.e. the solutions are flat connections). The non-trivial dynamics emerges through a projection, rather in the same way that solutions of the self-dual Yang-Mills equations emerge from a projection in twistor or harmonic space. The gauge group of this Chern-Simons theory contains the local Lorentz group, but is larger; in two dimensions it is just $\operatorname{sl}(2, R)$, and we shall show that it is this enhanced gauge group that yields Polyakov's current algebra. This gauge theoretic formulation of two-dimensional gravity has received some attention in the literature, but is in fact a two-dimensional version of a four-dimensional construction first described in a little noticed paper of Pagels [3], in which some of the basic ideas of topological field theory [4] were introduced. Related ideas can also be found in [5]. In fact, Pagels' construction of general relativity is trivially extendable to any dimension. 


\section{Two-dimensional gravity as gauge theory.}

$$
\begin{aligned}
& \text { scalar } R \\
& \qquad R_{\mu \nu \rho \sigma}=\frac{1}{2} R\left(g_{\mu \rho} g_{\nu \sigma}-g_{\mu \sigma} g_{\nu \rho}\right)
\end{aligned}
$$

In two dimensions the curvature tensor $R_{\mu \nu \rho \sigma}$ may be written in terms of the curvature

so that $R$ alone completely characterizes the local geometry. The relationship between the Ricci tensor $R_{\mu \nu}$ and $R$ is such that the Einstein tensor vanishes identically. The fact that Einstein gravity is inappropriate in two dimensions could also be deduced from the fact that the curvature term in the Hilbert-Einstein action is the Euler characteristic class for the manifold, which is independent of the metric. Because the full information on spacetime geometry is contained in the Ricci scalar $R$, Jackiw [6] and Teitelboim [7] proposed as an equation of motion $R=\Lambda$. This equation is derived from the following action [6]

$$
S=\int d^{2} x \sqrt{g} N(R-\Lambda)
$$

where $N$ is an auxiliary scalar field. By varying this action with respect to $N$ and $g_{\mu \nu}$ we recover the equations of motion

$$
R=\Lambda, \quad\left(\nabla_{\mu} \nabla_{\nu}-g_{\mu \nu} \nabla^{2}\right) N+\frac{1}{2} g_{\mu \nu} \Lambda N=0
$$

Notice that the equation $R=\Lambda$ does not involve $N$, this equation determines the metric, while the other one determines $N$ with no further restriction on $g_{\mu \nu}$. Despite appearances, this model can be written as a $\operatorname{sl}(2, R)$ gauge theory [8], in the same way that Pagels wrote a gauge theoretic action for four dimensional general relativity ( See also [9] for a gauge theoretical formulation of the dilaton gravity). In fact Pagels' formulation may be extended to any number of dimensions. We adopt the following conventions: Greek indices $\mu=0 \ldots d-1$ refer to space-time; upper case latin $A$ to an $S O(d, 1)$ gauge group and lower case latin $a=0, \ldots, d-1$ to the local Lorentz group, which is an $S O(d-1,1)$ subgroup of the full $S O(d, 1)$ gauge group. We introduce $S O(d, 1)$ gauge fields $\omega_{\mu}^{A B}=-\omega_{\mu}^{B A}$ which transform as the adjoint representation of $S O(d, 1)$ and a scalar field in the fundamental representation, $\phi^{A}$. The action, according to Pagels, is

$$
S=\int d^{d} x \alpha \epsilon^{A \ldots D E F} D \phi^{A} \ldots R^{D E} \phi^{F}+\beta D \phi^{A} \ldots D \phi^{E} \phi^{F} .
$$

Here, the elipsis refers to the appropriate number of $D \phi$ 's to soak up the indices on the $S O(d, 1) \epsilon$ symbol, $D \phi^{A}$ is the covariant exterior derivative of the zero-form $\phi^{A}$, and $R^{A B}$ is the curvature two-form of the $S O(d, 1)$ connection. Identifying the gravitational vielbein with the part of $D \phi^{A}$ transverse to $\phi^{A}$, the spin-connection with a similar transverse piece 
of the $S O(d, 1)$ connection and imposing the condition $\phi^{2}=M^{2}$ we recover the HilbertEinstein action with non-zero cosmological constant. In two-dimensions this procedure yields a total divergence. Instead we shall use a variant of this action

$$
S=\int d^{2} x \epsilon^{\mu \nu} \epsilon^{A B C} R_{\mu \nu}^{A B} \phi^{C}
$$

where the gauge field strength is given by $R_{\mu \nu}^{A B}=\partial_{[\mu} \omega_{\nu]}^{A B}+\omega_{[\mu}^{A C} \omega_{\nu]}^{C B}$ and $\epsilon^{A B C}$ is the antisymmetric $\operatorname{sl}(2, R)$ symbol.

The three components of the $s l(2, R)$ gauge fields $\omega_{\mu}^{A B}$ will eventually be identified as the spin connection $\omega_{\mu}^{a b}$ and the two components of the veirbein $\omega_{\mu}^{a 3}=e_{\mu}^{a}$. The equations of motion follow by varying the action with respect to the dynamical degrees of freedom $\omega_{\mu}^{A B}$ and $\phi^{A}$

$$
R^{A B}=0 \quad D \phi^{C}=0
$$

where $D \phi^{A}=\partial \phi^{A}+\omega^{A B} \phi^{B}$ is the covariant $s l(2, R)$ derivative. The general solution to the first equation can be written locally as $\omega^{A B}=\left(g^{-1} d g\right)^{A B}=\left(g^{-1}\right)^{A B}(d g)^{C B}$ where $g^{A B} \in \operatorname{sl}(2, R)$. Then the solution to the second equation can be written as $\phi^{C}=$ $\left(g^{-1}\right)^{C D} \phi_{0}^{D}$, where $\phi_{0}$ are constants. We observe that the space of all solutions of the classical equations of motion modulo gauge transformations is finite dimensional. Thus the action (2.5) describes a topological field theory.

In order to make contact with two-dimensional gravity we make the following identifications for the gauge fields: $\omega_{\mu}^{a 3}=s^{-1} e_{\mu}^{a}$, where $e_{\mu}^{a} a=1,2$ are the two components of the veirbein and $\omega_{\mu}^{a b}=\omega_{\mu}$ is the spin connection. The introduction of the arbitrary scale parameter $s$ is necessary because while the veirbein is dimensionless, the gauge field has dimension one. Then the equations $R_{\mu \nu}^{a 3}=0$ provide the vanishing of torsion and establish the relation between the veirbein $e_{\mu}^{a}$ and the spin connection $\omega_{\mu}, \omega_{\mu}=-e^{-1} \epsilon^{\mu \nu} \partial_{\nu} e_{\rho}^{a} e_{a \mu}$. The remaining equation gives us $R=\Lambda$ with the identification $\Lambda=s^{-2}$. Finally combining the two equations for $\phi^{a}$, we can write the equation for $\phi^{3}$ as follows

$$
\left(\nabla_{a} \nabla_{b}-g_{a b} \nabla^{2}\right) \phi^{3}+\frac{1}{2} s^{-2} g_{a b} \phi^{3}=0
$$

So by identifying $\phi^{3}$ with $N$ we have shown that the $\operatorname{sl}(2, R)$ gauge theory classically is equivalent to the theory which was proposed by Jackiw [6] to describe two-dimensional gravity. 


\section{3. $\operatorname{sl}(2, R)$ Gauge.}

The variation of $e_{\mu}^{A}$ under a $s l(2, R)$ gauge transformation is

$$
\delta e_{\mu}^{A}=-D_{\mu} \rho^{A}=-\partial_{\mu} \rho^{A}-\epsilon^{A B C} e_{\mu}^{B} \rho^{C}
$$

where $\rho^{A}$ is the parameter of transformation. Under a diffeomorphism generated by a vector field $-v^{\kappa}$, the standard transformation law would be

$$
\delta e_{\mu}^{A}=-v^{\kappa} \partial_{\kappa} e_{\mu}^{A}-\partial_{\mu} v^{\kappa} e_{\kappa}^{A} .
$$

The two-dimensional metric is $g_{\mu \nu}=e_{\mu}^{a} e_{\nu}^{b} \eta_{a b}$, since we have identified $e_{\mu}^{a}$ with the veirbein. We choose light-cone coordinates and gauge fix in the following manner: $e_{-}^{-}=0, e_{+}^{+}=\frac{h_{++}}{\lambda}$ $e_{+}^{-}=\frac{1}{\lambda}, e_{-}^{+}=-\frac{1}{2} \exp (\phi) \lambda$ where $\phi(x), h_{++}(x)$ and $\lambda(x)$ are arbitrary functions. With this particular choice, the metric becomes

$$
g_{--}=0, \quad g_{++}=h_{++} \exp \phi, \quad g_{+-}=g_{-+}=\frac{1}{2} \exp \phi .
$$

This particular gauge was introduced in [10] in order to investigate the symmetry structure of two-dimensional gravity. Initially we have treated the veirbein $e_{\mu}^{a}$ and the spin connection $\omega_{\mu}^{a b}$ as independent variables. The equations of motion (2.6) provide a relation between them. In the gauge we have chosen, with the particular choice for the components of the veirbein, the two components of the spin-connection read

$$
\omega_{+}=-\partial_{-} h_{++}-\left(\partial_{-} \phi\right) h_{++}+\lambda \partial_{+}\left(\frac{1}{\lambda}\right), \quad \omega_{-}=-\partial_{-} \phi-\frac{\partial_{-} \lambda}{\lambda} .
$$

If we substitute these values into the equation $R_{\mu \nu}^{a b}=0$, we find the following equation of motion for the dynamical degrees of freedom $h_{++}(x)$ and $\phi(x)$ of the metric

$$
\partial_{+} \partial_{-} \phi+\frac{\Lambda}{2} \exp \phi=\partial_{-}^{2} h_{++}+\left(\partial_{-}^{2} \phi\right) h_{++}+\left(\partial_{-} \phi\right)\left(\partial_{-} h_{++}\right) .
$$

Under a gauge transformation the veirbein (gauge field) $e_{-}^{-}$transforms as $\delta e_{-}^{-}=-\partial_{-} \rho^{-}+$ $e_{-}^{-} \rho^{3}-\omega_{-} \rho^{-}$. Then the residual $\operatorname{sl}(2, R)$ gauge transformations correspond to those ones which respect this particular gauge choice, namely the transformations which satisfy $\delta e_{-}^{-}=$ 0 . The solution to this equation constrains $\rho^{-}$in the form $\rho^{-}=-e_{+}^{-} \tau\left(x^{+}\right)$, with $\tau\left(x^{+}\right)$ being an arbitrary function of $x^{+}$. As a result all gauge transformations generated by $\rho^{+}, \rho^{3}$ and $\rho^{-}=-e_{+}^{-} \tau\left(x^{+}\right)$respect this particular gauge choice. Let us proceed and calculate the variation of the physical degrees of freedom $\delta g_{++}$and $\delta g_{+-}$under residual gauge transformations. We find that

$$
\begin{aligned}
\delta g_{+-}=-\delta e_{+}^{-} e_{-}^{+}-e_{+}^{-} \delta e_{-}^{+} & =\frac{1}{2} e^{\phi} \delta \phi=\frac{1}{2} e^{\phi} \partial_{+} \tau\left(x^{+}\right)+\frac{1}{2} e^{\phi}\left(\partial_{+} \phi\right) \tau\left(x^{+}\right) \\
& -\frac{1}{2} e^{\phi}\left(\partial_{-} h_{++}\right) \tau\left(x^{+}\right)-\frac{1}{2} e^{\phi}\left(\partial_{-} \phi\right) h_{++} \tau\left(x^{+}\right)-\partial_{-}\left(e_{+}^{-} \rho^{+}\right) .
\end{aligned}
$$


Similarly we find that

$$
\begin{aligned}
& \delta g_{++}=-2 \delta e_{+}^{+} e_{+}^{-}-2 e_{+}^{+} \delta e_{+}^{-}=e^{\phi} \delta \phi h_{++}+e^{\phi} \delta h_{++}= \\
& -2 \partial_{+}\left(e_{+}^{-} \rho^{+}\right)-\lambda e^{\phi}\left(\partial_{+} \phi\right) \rho^{+}+\lambda e^{\phi}\left(\partial_{-} \phi\right) h_{++} \rho^{+}+\lambda e^{\phi}\left(\partial_{-} h_{++}\right) \rho^{+} \\
& +e^{\phi} h_{++} \partial_{+} \tau\left(x^{+}\right)+e^{\phi} h_{++} \partial_{+} \phi \tau\left(x^{+}\right)-e^{\phi} h_{++} \partial_{-} h_{++} \tau\left(x^{+}\right)-e^{\phi} h_{++}^{2} \partial_{-} \phi \tau\left(x^{+}\right) .
\end{aligned}
$$

We can now further fix the gauge by selecting a specific form for $e^{\phi}$. We choose $e^{\phi}=$ $\frac{1}{\left(\alpha\left(x^{+}\right) x^{-}+\beta\left(x^{+}\right)\right)^{2}}$. We seek the gauge transformations which preserve this form of $e^{\phi}$, namely we permit the arbitrary functions $\alpha$ and $\beta$ to vary in such a manner that preserve the form of $e^{\phi}$. This will determine $\rho^{+}$in terms of three unknown functions $\eta\left(x^{+}\right), \delta \alpha\left(x^{+}\right)$ and $\delta \beta\left(x^{+}\right)$as follows

$$
\rho^{+}=-e_{-}^{+} \frac{\delta \alpha}{\beta}\left(x^{-}\right)^{2}-e_{-}^{+} \frac{\alpha \delta \beta}{\beta^{2}}\left(x^{-}\right)^{2}-e_{-}^{+} \frac{\delta \beta}{\beta} x^{-}-e_{-}^{+} \eta \alpha^{2}\left(x^{-}\right)^{2}-2 e_{-}^{+} \eta \alpha \beta x^{-}-e_{-}^{+} \beta^{2} \eta .
$$

We have omitted the transformation which is generated by $\tau\left(x^{+}\right)$for simplicity. Having determined the form of $\rho^{+}$we will now determine the effect of the transformations generated by $\eta\left(x^{+}\right), \delta \alpha\left(x^{+}\right)$and $\delta \beta\left(x^{+}\right)$on the only remaining degree of freedom $h_{++}$. The solution to $(3.5)$ can be written as

$$
h_{++}\left(x^{+}, x^{-}\right)=\sqrt{2} J^{(+)}\left(x^{+}\right)-2 J^{(0)}\left(x^{+}\right) x^{-}+\sqrt{2} J^{(-)}\left(x^{+}\right)\left(x^{-}\right)^{2}
$$

where $J^{(-)}=-2 \partial_{+} \alpha+2 \frac{\alpha \partial_{+} \beta}{\beta}+\frac{\Lambda}{2}, J^{(+)}$and $J^{(0)}$ are arbitrary functions of $x^{+}$. By substituting (3.9) and (3.8) into (3.7) we find that $J^{(-)}, J^{(+)}$and $J^{(0)}$ transform as follows under the residual $\operatorname{sl}(2, R)$ gauge transformations

$\delta J^{(+)}=-\frac{1}{\sqrt{2}} \partial_{+}\left(\eta \beta^{2}\right)+\sqrt{2} \eta \beta^{2} J^{(0)}+2\left(\eta \alpha \beta+\frac{\delta \beta}{\beta}\right) J^{(+)}$

$\delta J^{(0)}=-\partial_{+}\left(\eta \alpha \beta+\frac{\delta \beta}{\beta}\right)+\sqrt{2} \eta \beta^{2} J^{(-)}-\sqrt{2}\left(\frac{\delta \alpha}{\beta}+\frac{\delta \beta \alpha}{\beta^{2}}+\eta \alpha^{2}\right) J^{(+)}$

$\delta J^{(-)}=-\frac{1}{\sqrt{2}} \partial_{+}\left(\eta \alpha^{2}+\frac{\delta \alpha}{\beta}+\frac{\delta \beta \alpha}{\beta^{2}}\right)-\sqrt{2}\left(\frac{\delta \alpha}{\beta}+\frac{\delta \beta \alpha}{\beta^{2}}+\eta \alpha^{2}\right) J^{(0)}-2\left(\eta \alpha \beta+\frac{\delta \beta}{\beta}\right) J^{(-)}(3$

This is the main result of the paper. The $J^{(+)}, J^{(-)}$and $J^{(0)}$ transform under the residual $s l(2, R)$ gauge transformations as the adjoint of an $s l(2, R)$ Kac-Moody algebra. The parameters of transformations $\epsilon^{+}=\frac{1}{\sqrt{2}}\left(\eta \beta^{2}\right), \epsilon^{0}=\left(\eta \alpha \beta+\frac{\delta \beta}{\beta}\right)$ and $\epsilon^{-}=\frac{1}{\sqrt{2}}\left(\eta \alpha^{2}+\frac{\delta \alpha}{\beta}+\frac{\delta \beta \alpha}{\beta^{2}}\right)$ are part of the original $\operatorname{sl}(2, R)$ gauge transformations with $\rho^{+}$constrained as in Eqn. (3.8). The Polyakov $\operatorname{sl}(2, R)$ Kac-Moody algebra in light cone gauge is a remnant of an $\operatorname{sl}(2, R)$ Kac-Moody symmetry algebra which manifests itself as a residual symmetry algebra in the $s l(2, R)$ gauge and it is related to the original $s l(2, R)$ tangent space gauge symmetry. Upon gauge fixing the original gauge transformations behave as coordinate transformations. In the next section we will investigate the relation of the $\operatorname{sl}(2, R)$ gauge to the light-cone and to the conformal gauge. 


\section{Relation to other Gauges.}

Polyakov has proposed to study two-dimensional gravity in the light-cone gauge where the line element is

$$
d s^{2}=d x^{+} d x^{-}+g_{++}(x)\left(d x^{+}\right)^{2} .
$$

The light-cone gauge is recovered by setting $\phi=0$ in (3.3). Then the metric takes the following form:

$$
g_{++}=h_{++}(x), \quad g_{--}=0, \quad g_{+-}=g_{-+}=\frac{1}{2}
$$

which is exactly the metric in the light cone gauge. The condition that the residual gauge transformations respect the gauge choice $\delta e_{-}^{-}=0$ and $\delta g_{+_{-}}=0$ provide the following constraints on $\rho^{+}$and $\rho^{-}$

$$
\rho^{-}=-e_{-}^{+} \tau\left(x^{+}\right), \quad \rho^{+}=-e_{-}^{+} h_{++} \tau\left(x^{+}\right)+e_{-}^{+} \partial_{+} \tau\left(x^{+}\right) x^{-}-e_{-}^{+} \eta\left(x^{+}\right) .
$$

We notice that $\rho^{+}$is further constrained than before Eqn. (3.8). This particular relation can also be recovered from (3.8) by setting $\alpha=\delta \alpha=\delta \beta=0$ and $\beta=1$.

In light-cone gauge the equation of motion for $h_{++}$becomes $\partial_{-}^{2} h_{++}(x)=\frac{\Lambda}{2}$. This can be seen by either setting $\phi=0$ into (3.5) or by substituting the values for the components of the spin connection in light-cone gauge

$$
\omega_{+}=-\partial_{-} h_{++}+\lambda \partial_{+}\left(\frac{1}{\lambda}\right), \quad \omega_{-}=-\frac{\partial_{-} \lambda}{\lambda}
$$

into (2.6). The solution to the equation of motion for the gravitational degree of freedom $h_{++}\left(x^{+}, x^{-}\right)$can be parametrised again in the following form

$$
g_{++}=h_{++}\left(x^{+}, x^{-}\right)=\sqrt{2} J^{(+)}\left(x^{+}\right)-2 J^{(0)}\left(x^{+}\right) x^{-}+\sqrt{2} J^{(-)}\left(x^{+}\right)\left(x^{-}\right)^{2}
$$

where $J^{(-)}=\frac{\Lambda}{2}$. The residual gauge transformations, Eqn. (4.3), act on the gravitational degree of freedom $h_{++}(x)$ as follows

$$
\begin{array}{r}
\delta h_{++}(x)=-\partial_{+} h_{++}(x) \tau\left(x^{+}\right)-\partial_{+} \tau\left(x^{+}\right)\left(2-x^{-} \partial_{-}\right) h_{++}(x) \\
+\partial_{+}^{2} \tau\left(x^{+}\right) x^{-}-\partial_{+} \eta\left(x^{+}\right)-\eta\left(x^{+}\right) h_{++}(x) .
\end{array}
$$

This permits us to calculate the variations of the currents $J^{(+)}\left(x^{+}\right), J^{(-)}\left(x^{+}\right)$and $J^{(0)}\left(x^{+}\right)$ under the residual gauge transformations generated by $\eta\left(x^{+}\right)$

$$
\delta J^{(+)}=-\frac{1}{\sqrt{2}} \partial_{+} \eta\left(x^{+}\right)+\sqrt{2} J^{(0)} \eta\left(x^{+}\right), \quad \delta J^{(0)}=\sqrt{2} J^{(-)} \eta\left(x^{+}\right), \quad \delta J^{(-)}=0 .
$$

As we have further fixed our $\operatorname{sl}(2, R)$ gauge in order to recover the light-cone gauge, only part of the $\operatorname{sl}(2, R)$ Kac-Moody remains as a residual symmetry, more specifically only the 
transformations generated by $\eta\left(x^{+}\right)$respect the light cone gauge. Let's now demonstrate that residual gauge transformations act on $h_{++}(x)$ as residual diffeomorphisms. The latter are generated by a vector field of the form

$$
v^{+}=v^{+}\left(x^{+}\right), \quad v^{-}=-\left(\partial_{+} v^{+}\right) x^{-}+g\left(x^{+}\right)
$$

This is the form of the diffeomorphisms that preserve the light-cone gauge. Under these transformations $h_{++}$transforms as

$$
\begin{array}{r}
\delta h_{++}(x)=-\partial_{+} h_{++}(x) v^{+}\left(x^{+}\right)-\partial_{+} v^{+}\left(x^{+}\right)\left(2-x^{-} \partial_{-}\right) h_{++}(x) \\
+\partial_{+}^{2} v^{+}\left(x^{+}\right) x^{-}-\partial_{+} g\left(x^{+}\right)-g\left(x^{+}\right) h_{++}(x)
\end{array}
$$

which is the same as Eqn. (4.6).

We briefly comment on the relation of the $s l(2, R)$ gauge and the conformal gauge. By setting $h_{++}=0$ in (3.3) we recover the conformal gauge $g_{++}=g_{--}=0$ and $g_{+-}=g_{-+}=$ $\frac{1}{2} e^{\phi}$. The equation of motion (3.5) becomes $\partial_{+} \partial_{-} \phi+\frac{\Lambda}{2} e^{\phi}=0$ which we recognise as the Liouville equation. The residual gauge transformations which respect this particular form follow by simply demanding $\delta h_{++}=0$. This will suplement the relation $\rho^{-}=e_{+}^{-} \tau\left(x^{+}\right)$ which follows from $\delta e_{-}^{-}=0$ with $\rho^{+}=e_{-}^{+} \sigma\left(x^{-}\right)$. The residual gauge transformations generated by $\rho^{+}$and $\rho^{-}$act on $g_{+-}$as diffeomorphisms generated by $\left(\tau\left(x^{+}\right), \sigma\left(x^{-}\right)\right)$since under those $g_{+-}$transforms as $\delta g_{+-}=-\partial_{+} \tau-\partial_{-} \sigma$. This is identical to the action of residual diffeomorphisms in the conformal gauge.

\section{Conclusions.}

Let us review the results presented in this paper. Two-dimensional gravity exhibits a $s l(2, R)$ current algebra in the light-cone gauge. It is the presence of this algebra that makes the theory tractable although it's origin appears mysterious. In this paper we offered an explanation about the origin of this important algebra. The point is that two-dimensional gravity can be formulated as a $s l(2, R)$ gauge theory. Upon gauge fixing the current algebra manifests itself as a residual symmetry algebra. Further gauge fixing to light cone gauge restricts the gauge freedom and only part of the current algebra survives as a residual symmetry. The residual gauge transformations act on the gravitational degrees of freedom as residual diffeomorphisms [11].

\section{Acknowledgments.}

I would like to thank A. Chamseddine and M. Evans for useful discussions. This work was supported in part by the Department of Energy Contract Number DE-FG0291ER40651-TASKB. 


\section{References.}

[1] A. M. Polyakov Mod. Phys. Lett. A2 (1987), 893.

[2] M. Bershadsky and H. Ooguri, Commun. Math. Phys. 126 (1989), 49; A. Alekseev and S. Shatashvili, Nucl. Phys. B323 (1989), 719; A. Chamseddine and M. Reuter, Nucl. Phys. B317 (1989), 757; E. Argyres, C. Papadopoulos and E. Floratos Phys. Lett. 234B (1990), 304; J. Helayel-Neto, S. Mokhtari and A. Smith, Phys. Lett. 236B (1990), 12; E. Egorian and R. Manvelian, Mod. Phys. Lett. A5 (1990), 2371; E. Abdalla, M. Abdalla, J. Gamboa and A. Zadra, Phys. Lett. 273B (1991), 222; A. Smallagic and E. Spallucci, Phys. Lett. 317B (1993), 526; P. Forgacs, A. Wipf, J. Balog, L. Feher and L. O’ Raifeartaigh, Phys. Lett. 227B (1989), 214; P. Schaller and T. Strobl, Phys. Lett. 337B (1994), 266; N. Mohammedi, Mod. Phys. Lett. A5 (1990), 1251.

[3] H. Pagels, Phys. Rev. D29 (1984), 1690.

[4] E. Witten, Commun. Math. Phys. 117 (1988), 353; Nucl. Phys. B311 (1988), 46.

[5] A. H. Chamseddine and P. C. West, Nucl. Phys. B129 (1977), 39; A. H. Chamseddine, Ann. Phys. (NY) 113 (1978), 212; S. MacDowell and F. Mansouri, Phys. Rev. Lett. 38 (1977), 739.

[6] R. Jackiw Quantum Theory of Gravity ed. S. Christensen (Adam Hilger, Bristol) (1984).

[7] C. Teitelboim Quantum Theory of Gravity ed. S. Christensen (Adam Hilger, Bristol) (1984).

[8] T. Fukuyama and K. Kamimura, Phys. Lett. 160,B (1985), 259; M. Awada and A. H. Chamseddine, Phys. Lett. 233B (1989), 79; A. H. Chamseddine and D. Wyler, Phys. Lett. 228B (1989), 75; Nucl. Phys. B340 (1990), 595; K. Isler and C. Trugenberger, Phys. Rev. Lett. 63 (1989), 834; H. Terao, Nucl. Phys. B395 (1993), 623.

[9] H. Verlinde in The Sixth Marcel Grossman Meeting on General Relativity, H. Sato ed. (World Scientific, 1992); D. Cangemi and R. Jackiw, Phys. Rev. Lett. 69 (1992), 233; Phys. Lett. 299B (1993), 24; D. Cangemi, R. Jackiw and B. Zwiebach, Ann. Phys. (NY) 245 (1996), 408.

[10] K.-W. Xu and C.-J. Zhu Int. Jour. Mod. Phys. A6 (1991), 2331.

[11] A. M. Polyakov, Int. Jour. Mod. Phys. A5 (1990), 833. 